\title{
Effect of dipolar interactions on the magnetization of a cubic array of nanomagnets
}

\author{
Marisol Alcántara Ortigoza Richard A. Klemm $₫$ and Talat S. Rahmant \\ Department of Physics, Kansas State University, Manhattan, KS 66506 USA
}

(Dated: October 7, 2018)

\begin{abstract}
We investigated the effect of intermolecular dipolar interactions on an ensemble of 100 3D-systems of $5 \times 5 \times 4$ nanomagnets, each with spin $S=5$, arranged in a cubic lattice. We employed the LandauLifshitz-Gilbert equation to solve for the magnetization curves for several values of the damping constant, the induction sweep rate, the lattice constant, the temperature, and the magnetic anisotropy. We find that the smaller the damping constant, the stronger the maximum induction required to produce hysteresis. The shape of the hysteresis loops also depends on the damping constant. We find further that the system magnetizes and demagnetizes at decreasing magnetic field strengths with decreasing sweep rates, resulting in smaller hysteresis loops. Variations of the lattice constant within realistic values $(1.5 \mathrm{~nm}-2.5 \mathrm{~nm})$ show that the dipolar interaction plays an important role in the magnetic hysteresis by controlling the relaxation process. The temperature dependencies of the damping constant and of the magnetization are presented and discussed with regard to recent experimental data on nanomagnets. Magnetic anisotropy enhances the size of the hysteresis loops for external fields parallel to the anisotropy axis, but decreases it for perpendicular external fields. Finally, we reproduce and test a previously reported magnetization curve for a 2D-system [M. Kayali and W. Saslow, Phys. Rev. B 70, 174404 (2004)]. We show that its hysteretic behavior is only weakly dependent on the shape anisotropy field and the sweep rate, but depends sensitively upon the dipolar interactions. Although in 3D systems, dipole-dipole interactions generally diminish the hysteresis, in two-dimensional systems, they strongly enhance it. For both square two-dimensional and rectangular three-dimensional lattices with $\boldsymbol{B} \|(\hat{\boldsymbol{x}}+\hat{\boldsymbol{y}})$, dipole-dipole interactions can cause large jumps in the magnetization.

PACS numbers: 75.40.Mg, 75.60.Ej, 75.75.+a, 75.50.Xx
\end{abstract}

\section{INTRODUCTION}

The need of smaller memory storage devices, 1, 2, 3, 4, 5, 6, 7, 8, 9, 10, 11, 12, 13, 14 the interest in developing quantum computing, 15$]$ and the hope for understanding the relationship between the macroscopic and microscopic magnetic behaviors has led intense research into the properties of nanoscale magnets. 1, 2, 3, 4, 5, 6, 7, 8, 9, 10, 11, 12, 13, 14, 15, 16, 17, 18, 19, 20, 21, 22, 23, 24, 25, 26, 27, 28, 29, 30, 31, 32] Many issues still remain unclear and serious problems must be overcome in order for them to be technologically useful. Prominent among these is the loss of memory during magnetic relaxation.

Ferromagnetic nanodots are complex systems consisting of up to hundreds of magnetic atoms within a single dot. [5, 11, 12] In this case, interparticle interactions along with anisotropy effects dominate the dynamics of the systems, and control the magnetization processes. 8] Moreover, since interdot exchange interactions are negligibly small, the dynamics of the ferromagnetic nanodot arrangements are strongly influenced by dipolar interdot interactions. 13, 14]

Single molecule magnets (SMM's) consist of clusters of only a few magnetic ions, and are thus among the smallest and simplest nanomagnets, but are also wellcharacterized systems exhibiting magnetic hysteresis. 27. In SMM's, the one-body tunnel picture of the magnetization mostly explains this phenomenon in the sense that the sequence of discrete steps in those curves provides evidence for resonant coherent quantum tunneling. 28, 29, 30] Nevertheless, this one-body tunnel model neglects intermolecular interactions, and is not always sufficient to explain the measured tunnel transitions. 31, 32. A close examination of the magnetization curves reveals fine structures which cannot be explained by that model. Wernsdorfer et al. suggest that these additional steps are due to collective quantum processes, called spin-spin cross relaxation (SSCR), involving pairs of SMM's which are coupled by dipolar and/or exchange interactions. 31, 32] If dipolar and/or exchange interactions cooperate in the relaxation process, then one might hope to be able to better control such loss of magnetic memory.

Analyzing the relaxation of the magnetization is difficult for both SMM's and ferromagnetic nanodots. Besides dipolar interactions, many other factors may be involved in such processes. Geometric features, such as the shape and volume of the magnets, as well as the type of lattice on which they are placed, can directly influence the anisotropy barriers and the easy axis directions. In the case of SMM's, a quantum treatment has to be considered to show that resonant tunneling of the magnetization results in the discrete steps appearing in the low temperature $T$ magnetization curves. Although in many SMM's the intercluster exchange interactions are negligible, as for ferromagnetic nanodots, in other SMM's, such interactions are comparable in strength to the dipolar interactions. 32 Besides the quadratic Heisenberg and quadratic anisotropic intramolecular exchange interac- 
tions, some SMM's are thought to contain intramolecular interactions of the Dzyaloshinskii-Moriya type. 33. Additional higher order, anisotropic spin exchange interactions further complicate the problem. Therefore, by studying models that deal with each one of these factors separately, one hopes to simplify the problem, to build up gradually a more realistic system, and at the same time, to elucidate how each of these factors contributes to the magnetization process.

With regard to SMM's, there have been recent approaches to the quantum dynamics of the low- $T$ relaxation. 17, 34, 35, 36, 37, 38, 39] Prokof'ev and Stamp assumed a single relaxation mode, 34] in which the dipolar and hyperfine fields are frozen unless an SMM flips its spin. Then by assuming the effective field around each SMM is that of randomly placed dipoles, they obtained an expression for the low- $T$ decrease proportional to $t^{p}$ of the magnetization of each SMM from its fully magnetized state, 34 , 40, 41] where $p \approx 0.5-0.7$, but $p$ might be as large as $0.7 .34,35,36,37$. This procedure was restricted to very small deviations of the magnetization from its saturated value, so it is not useful for studying the central portion of the hysteresis curves, for which the magnetization can be small. Moreover, as first argued for ferromagnets by Anderson, 42] the spinspin and spin-lattice relaxation times can be very different, so that such simple behavior is not expected. In fact, experiments on SMM's have shown that an exponential relaxation of the magnetization is consistent with the data, 38, 39] so that as a minimum, one requires two distinct relaxation times for SMM's, which could be very different from one another. 42.

The most commonly studied model of spin dynamics containing two distinct relaxation parameters is the Landau-Lifshitz-Gilbert (LLG) equation. 43, 44] Using the LLG equation, Kayali and Saslow (KS) investigated the hysteresis curves at $T=0$ for two-dimensional (2D) square arrays of 4 to 169 ferromagnetic nanodots subject to dipole-dipole interactions and a magnetic field applied in various directions within the array's $x y$ plane. 45. They included anisotropy effects via an effective field proportional to the $z$-component of each dot's dipole moment. Earlier studies of square planar lattices of 9 to 36 ferromagnetic dots were made by Stamps and Camley. 46. In addition, Zhang and Fredkin (ZF) studied the LLG model to obtain the zero-field time decay of the easyaxis magnetization of a three-dimensional (3D) cubic lattice of $12 \times 12 \times 12$ Stoner-Wohlfarth particles interacting with each other via dipole-dipole interactions. 14] Since the size (or radius) of the Stoner-Wohlfarth particles was taken to be much less than the lattice constant, they could be treated as point-like magnetic moments, the classical analogue of SMM's.

Here we study only the effects of the intermolecular dipole-dipole interactions upon the magnetization curves for an ensemble of $N_{c}=1003 \mathrm{D}$ cubic crystals each containing $N=5 \times 5 \times 4$ nanomagnets, all with the same magnetic moment. As in the ZF model of StonerWohlfarth particles, we take the lattice parameter to be much greater than the nanomagnet size or radius. Except when a strong anisotropy field is present, we assume that there is no long-range order in the $T$ regime of interest, so that in the absence of an external magnetic field, the magnetization of each nanomagnet crystal is essentially zero. We note that long-range ordering was claimed to exist in such a system with Ising spin anisotropy. 47, 48] In our studies with a strong anisotropy field $\boldsymbol{H}_{A}$, hysteresis curves exhibiting a substantial zero-field magnetization were obtained for the applied magnetic induction $\boldsymbol{B} \| \boldsymbol{H}_{A}$ after the system had been fully magnetized by $\boldsymbol{B}$. The strength of the dipole interactions is primarily determined by the lattice constant, $a$, which we vary from 1.25 $\mathrm{nm}$ to $2.5 \mathrm{~nm}$. The dynamics of each nanomagnet are assumed to be given by the LLG equation, which includes precession and damping relaxation processes, the damping coefficient $\alpha$ of which can also depend upon $T$. 44, 50] Then, the magnetic moment $\boldsymbol{M}_{i}^{c}$ of the $i$ th nanomagnet within the $c$ th crystal of our ensemble obeys

$$
\begin{aligned}
\frac{d \boldsymbol{M}_{i}^{c}}{d t} & \equiv \gamma \boldsymbol{M}_{i}^{c} \times \boldsymbol{B}_{i}^{c, \text { eff }}-\frac{\alpha}{M_{s}} \boldsymbol{M}_{i}^{c} \times\left(\boldsymbol{M}_{i}^{c} \times \boldsymbol{B}_{i}^{c, \text { eff }}\right),(1) \\
\boldsymbol{B}_{i}^{c, \text { eff }} & =\boldsymbol{B}+\left(\boldsymbol{B}_{i}^{c}\right)_{\mathrm{dip}},
\end{aligned}
$$

where $\gamma=g \mu_{B}$ is the gyromagnetic ratio, $M_{s}=g \mu_{B} S$ is the magnetic moment of an individual nanomagnet, and $\left(\boldsymbol{B}_{i}^{c}\right)_{\text {dip }}$ is the contribution to the effective magnetic induction $\boldsymbol{B}_{i}^{c, \text { eff }}$ at the $i$ th nanomagnet within the $c$ th crystal arising from dipole-dipole interactions between it and the other nanomagnets within the same crystal,

$$
\left(\boldsymbol{B}_{i}^{c}\right)_{\mathrm{dip}}=\frac{\mu_{0}}{4 \pi} \sum_{j} \frac{3\left(\boldsymbol{M}_{j}^{c} \cdot \boldsymbol{r}_{i j}\right) \boldsymbol{r}_{i j}-r_{i j}^{2} \boldsymbol{M}_{j}^{c}}{r_{i j}^{5}},
$$

where the prime indicates that the $j=i$ term is omitted. The second term of Eq. (1), the damping term, was first introduced by Landau and Lifshitz [4] and later by Gilbert to give a phenomenological description of the relaxation of the magnetization. They did not derive it from first principles due to the enormous complexity of summarizing all of the relaxation processes into a single term. As noted above, when ferromagnetic interactions are present, $\alpha / \gamma$ is generally expected to be $\ll 1$. 42. By extending to electronic spin systems the WangsnessBloch model of nuclear spin magnetic relaxation by magnetic dipole coupling to a heat bath, [49] Fredkin and Ron showed that the damping term could be derived for large spin values and $\kappa=\hbar \gamma H / k_{B} T \ll 1$, where $\hbar$ and $k_{B}$ are Planck's constant divided by $2 \pi$ and Boltzmanns' constant, respectively, and in our case $H=B_{i}^{c, \text { eff }}$. [50] To the extent that electric quadrupole interactions could be neglected, $\alpha$ varies inversely with $T$ for $\kappa \ll 1$, but depends upon $\kappa$ otherwise. [50] 
More recently, a different derivation of the Gilbert damping term was derived from a spin Hamiltonian containing the interaction between the spin and the radiation field, which is induced by the precessing magnetization itself. 51, 52 In that case, no explicit $T$ dependence of $\alpha$ was given. We remark that rather complex explicit expressions for $\alpha$ for the different system of local spin moments arising from $p-d$ kinetic-exchange coupling of the itinerant-spin subsystem in ferromagnetic semiconductor alloys have been given recently. 53. In any event, the damping coefficient $\alpha$ at some $T$ value must be determined experimentally for each system.

In order to study the magnetization of ferromagnetic dots, KS used an extremely large value for the damping coefficient, $\alpha / \gamma=0.6$, a huge sweep rate, $\frac{\Delta B}{\Delta t} \sim 3000$ $\mathrm{T} / \mathrm{s}$, and a small maximum external induction $B_{\max }=$ $2 \mu_{0} M_{s}$. In our studies of nanomagnet arrays, we used values of $\alpha / \gamma$ that varied from these values to values 12 orders of magnitude smaller. Depending upon the $\alpha$ values, we also varied the sweep rate $\frac{\Delta B}{\Delta t}$ from those values to the the much smaller $\sim 10^{-3} \mathrm{~T} / \mathrm{s}$, and varied $B_{\max }$ from much larger values $(2 \mathrm{~T})$, comparable to those reported in SMM experiments, 23, 26] to those used by KS. Similarly, the lattice constants reported in the present work are in accordance with the near neighbor separation in the most extensively studied SMM crystals. Quantum processes within the individual SMM's will be treated in a separate publication. 54

The present paper is organized follows. In Sec. II, we present our model system and a brief description of the overall calculation procedure that we followed. In Sec. III, we solve the LLG for each nanomagnet subject to both the external and the combined dipolar inductions. In Sec. IV, we present and discuss our main results for the magnetization curves, which are evaluated at various values of the sweep rate, $T, a$, the anisotropy field, and $\alpha / \gamma$. When spin anisotropy is present, we study the cases $\boldsymbol{B} \| \boldsymbol{H}_{A}$ and $\boldsymbol{B} \perp \boldsymbol{H}_{A}$. In Sec. V, we reproduce one of the KS magnetization curves for square 2D lattices, and vary some of their parameters to show that the results are almost independent of the sweep rate over the range $\sim 300-6000 \mathrm{~T} / \mathrm{s}$. Analogously, we show that the anisotropy field does not affect significantly the magnetization curves until its magnitude is comparable to $B_{\max } / \mu_{0}$. By varying the lattice constant, we also show that the results of KS are very sensitive to the strength of the dipolar fields, which mainly govern the behavior of the magnetization of such systems. Finally, in Sec. VI, we summarize our main conclusions.

\section{MODEL SYSTEM}

In the present work we consider an ensemble of $N_{c}=$ 100 cubic crystals (or configurations), each configuration consisting of $N=5 \times 5 \times 4=100$ nanomagnets, each with ground state spin $S=5$, which interact with one another only via dipolar interactions. Each of the $N_{c}$ system configurations $c=1, \ldots, N_{c}$ is constructed to have a starting total magnetic moment $\boldsymbol{M}_{c} \approx 0$ at $\boldsymbol{B}=0$. The hysteresis curves are obtained for each configuration, and these are then averaged over the $N_{c}$ configurations. One then obtains the magnetization $\overrightarrow{\mathcal{M}}(B)$ curves, where $\overrightarrow{\mathcal{M}}=\left\langle\boldsymbol{M}_{c}\right\rangle_{c} / V$ is the configuration averaged magnetization, $V$ is the crystal volume, and $B=|\boldsymbol{B}|$.

\section{Ensemble of random spin configurations}

In order to proceed, we first find a large number $N_{c}$ of random spin configurations $c$ of $N=100$ spins, such that for each configuration, $\boldsymbol{M}_{c} / M_{s} \approx 0$ at $\boldsymbol{B}=0$ and as $T \rightarrow \infty$, where the total magnetic moment

$$
\boldsymbol{M}_{c}(t, \boldsymbol{B})=\sum_{i=1}^{N=100} \boldsymbol{M}_{i}^{c}(t, \boldsymbol{B}) .
$$

At the start of the iteration, we take $t=0, \boldsymbol{B}=0$, and $T \rightarrow \infty$ in the absence of the dipole-dipole (or any other inter-spin) interactions for configuration $c$. Then we select those configurations for which $\left|\boldsymbol{M}_{c}\right| / M_{s} \leq 0.1$, which we deem sufficiently close to $\boldsymbol{M}_{c} \approx 0$. Our resulting magnetization curves are based upon the average over $N_{c}=100$ configurations, each one containing $N=100$ similarly chosen nanomagnets.

We reiterate that $N$ is the number of nanomagnets in each configuration, and $N_{c}$ is the number of configurations studied. Although we have chosen both of these numbers to be 100 in order to obtain reliable statistics, $N$ and $N_{c}$ have completely different meanings. Finding many $\left(N_{c}\right)$ configurations, each of which has an almost vanishing initial magnetization consumes a significant amount of computer time, especially if the number $N$ of nanomagnets per configuration is not very large. However, choosing a rather small number $N$ of nanomagnets reduces the time required to calculate the dipolar field at each nanomagnet due to all of its neighbors, which must be calculated at each integration time step of the LLG equation, offsetting the large amount of computer time required to set up $N_{c}$ initially nearly-nonmagnetic configurations.

\section{Evolution of the magnetization versus field curves}

In this model one increases the external magnetic induction $\boldsymbol{B}$ in discrete steps $\boldsymbol{\Delta} \boldsymbol{B}$, until $\boldsymbol{B}=\boldsymbol{B}_{\max }$, where $B_{\max }=\left|\boldsymbol{B}_{\max }\right|$ has to be large enough to align every nanomagnet in its direction. How large $B_{\max }$ has to be generally depends upon $T$, the field sweep rate $\frac{\Delta B}{\Delta t} \equiv \frac{|\Delta B|}{\Delta+}$, the lattice parameter $a$, and the crystal structure. 27] In addition, the steps $|\Delta \boldsymbol{B}|$ must be small 
enough to give rise to numerically smooth $\mathcal{M}(B)$ curves. We therefore set $|\Delta \boldsymbol{B}|=B_{\max } / N_{B}$, where the number of steps $N_{B} \gg 1$ should be on the order of $10^{3}$. After each magnetic step, one allows each of the nanomagnets to relax for a fixed amount of time $\Delta t$, which is chosen to be sufficiently small that the nanomagnets do not reach equilibrium. Otherwise, in the absence of a sufficiently strong anisotropy field, no hysteresis would result.

First, we choose one of our configurations $c$ (e. g., $c=1$ ) and set the moments of the nanomagnets equal to their values in this initially nonmagnetic configuration, $\left\{\boldsymbol{M}_{i}^{c=1}(t=0, \boldsymbol{B}=0)\right\}_{i=1, \ldots, N}$. That is, just after we turn on the magnetic induction in the $x$ direction by the amount $\boldsymbol{B}=\Delta \boldsymbol{B}$, the nanomagnets have not yet precessed from their initial configuration. Then, we calculate the effective magnetic induction $\boldsymbol{B}_{i}^{c=1 \text {,eff }}$ at each of the $i=1, \ldots, N$ nanomagnets for $c=1$. To do so, we must calculate the dipolar induction in Eq. (3) due to the presence of all the other nanomagnets.

Then, we let each of the nanomagnets evolve in the presence of its effective magnetic induction for a chosen fixed time interval $\Delta t$. To do this accurately, we break $\Delta t$ up into $N_{t}$ intervals $d t=\Delta t / N_{t}$. Obviously, this is extremely time consuming, because it is necessary to recalculate the effective induction at each nanomagnet after each time-integration step of width $d t$. Once the whole set of moments $\left\{\boldsymbol{M}_{i}^{1}(t=\Delta t, \boldsymbol{B}=\Delta \boldsymbol{B})\right\}_{i=1, \ldots, N}$ is obtained, we proceed to calculate the configuration magnetic moment, $\boldsymbol{M}_{c=1}(\Delta t, \Delta \boldsymbol{B})$, for this choice of fixed sweep rate, $\Delta B / \Delta t$, from

$$
\begin{aligned}
\boldsymbol{M}_{c}(t, \boldsymbol{B}) & =\frac{\sum_{i=1}^{N} \boldsymbol{M}_{i}^{c}(t, \boldsymbol{B}) \exp \left[-\beta \mathcal{H}_{i}^{c}(t, \boldsymbol{B})\right]}{\sum_{i=1}^{N} \exp \left[-\beta \mathcal{H}_{i}^{c}(t, \boldsymbol{B})\right]}, \\
\mathcal{H}_{i}^{c}(t, \boldsymbol{B}) & =-\boldsymbol{B}_{i}^{c, \text { eff }}(t, \boldsymbol{B}) \cdot \boldsymbol{M}_{i}^{c}(t, \boldsymbol{B})
\end{aligned}
$$

by setting $c=1, t=\Delta t$ and $\boldsymbol{B}=\Delta \boldsymbol{B}$, where $\boldsymbol{B}_{i}^{c, \text { eff }}(t, \boldsymbol{B})$ is given from Eqs. (2) and (3), $\beta=1 / k_{B} T$, and $k_{B}$ is Boltzmann's constant. Since $\left(\boldsymbol{B}_{i}^{c}\right)_{\text {dip }}$ as given by Eq. (3) in $\boldsymbol{B}_{i}^{c, \text { eff }}(t, \boldsymbol{B})$ contains a self-fieldless single sum, there is no site overcounting in Eq. (6).

We are interested in $\boldsymbol{M}_{c}\left(\boldsymbol{B}, \frac{\Delta B}{\Delta t}\right)$, a thermodynamic quantity that does not explicitly depend upon $t$. Although the $\boldsymbol{M}_{i}^{c}$ and $\boldsymbol{B}_{i}^{c, \text { eff }}$ for each nanomagnet are evaluated at each time step $d t$, the statistical weighting in Eqs. (15) and (6) is only evaluated at the end of each fixed interval $\Delta t$, which has a one-to-one correspondence with $\Delta B$. Thus, this single-configuration average can be directly compared to those in different configurations after the same number of intervals. Moreover, since $\Delta t=\Delta B\left(\frac{\Delta B}{\Delta t}\right)^{-1}, \boldsymbol{M}_{c}(t, \boldsymbol{B})$ for our purpose can be written as $\boldsymbol{M}_{c}\left(\frac{\boldsymbol{B}}{\Delta B / \Delta t}, \boldsymbol{B}\right)$, which is effectively a function of $\boldsymbol{B}$ and $\frac{\Delta B}{\Delta t}$.

Next, we increase the external magnetic induction by another equal step $\boldsymbol{\Delta} \boldsymbol{B}$, and let the nanomagnets precess during another equal time interval, $\Delta t$, under the action of the new effective induction. We continued increasing $\boldsymbol{B}$ in this equal step fashion a total of $N_{B}$ times, until $\boldsymbol{B}=\boldsymbol{B}_{\max }$. At this point, the incremental induction direction is reversed, setting $\boldsymbol{B}=\boldsymbol{B}_{\max }-\Delta \boldsymbol{B}$ for the same time interval $\Delta t$, repeating the procedure $2 N_{B}$ times, until $\boldsymbol{B}=-\boldsymbol{B}_{\max }$. After that, we reverse the incremental induction direction once again, setting $\boldsymbol{B}=-\boldsymbol{B}_{\max }+\Delta \boldsymbol{B}$ for the same time interval $\Delta t$, etc., and continue $N_{B}$ times until $\boldsymbol{B}=0$ is reached, or until the configuration magnetization hysteretic loop (if it exists) is closed. Then, one repeats the entire procedure above described for each of the other $N_{c}-1$ configurations $c=2, \ldots, N_{c}$, keeping the time intervals $\Delta t$ and the subintervals $d t$ constant for each step in each configuration. Once all of the calculations for each of the $N_{c}$ configuration are finished, we average the results over the $N_{c}$ configurations, obtaining,

$$
\left\langle\boldsymbol{M}_{c}\left(\frac{\mathbf{B}}{\Delta B / \Delta t}, \boldsymbol{B}\right)\right\rangle_{c}=\frac{1}{N_{c}} \sum_{c=1}^{N_{c}} \boldsymbol{M}_{c}\left(\frac{\boldsymbol{B}}{\Delta B / \Delta t}, \boldsymbol{B}\right) .
$$

Then, the magnetization $\overrightarrow{\mathcal{M}}$ is easily calculated. Having tabulated $\overrightarrow{\mathcal{M}}$ for every external magnetic induction step with fixed $\frac{\Delta B}{\Delta t}, T, a, M_{s}, \alpha$, and $B_{\max }$, we generate the magnetization curve $\mathcal{M}(B)$ for this set of parameters.

\section{Variation of experimental parameters}

Unlike the parameters such as $B_{\max }$ and $d t$, which are details of the theoretical calculation, other parameters can in principle be varied in experiments in a variety of materials. Using the same initial dipole configurations we repeat the whole procedure with different values of $\alpha$, $\frac{\Delta B}{\Delta t}, T$, and $a$. The only parameters that can be experimentally varied in studies on a particular sample are $\frac{\Delta B}{\Delta t}$ and $T$, since the other parameters are fixed. Nevertheless, the possibility of setting the nanomagnets further apart by varying the composition of the non-magnetic ligand groups in SMM's, for example, justifies the study of the variation of $a$. Also, given that the damping term appearing in the LLG equation is phenomenological, and that in most cases $\alpha$ should be determined experimentally, we have also examined its variation. We note that $\alpha$ is expected to depend inversely upon $T$, unless $T$ is sufficiently low that thermal processes no longer dominate the relaxation. 14 We keep $M_{s}$ fixed.

\section{INTEGRATION OF THE LLG EQUATION FOR ONE NANOMAGNET}

The magnetic moment of each nanomagnet is obtained by numerically integrating the LLG equation. The timeevolution of one nanomagnet must be determined synchronously with all its neighbors in order to calculate 
the dipolar induction acting on each of them at a given time. To solve the LLG equation for the $i$ th nanomagnet in the $c$ th crystal, we first rotate its coordinates at each time integration step such that $\boldsymbol{B}_{i}^{c, \text { eff }}(t) \| \hat{\boldsymbol{z}}(t)$. We then solve the resulting differential equations for either the coordinate spherical angles $\theta(t), \phi(t)$, or the components of $\boldsymbol{M}_{i}^{c}(t)$, as shown in Appendix A. The quantity relevant to each spherical angle or component of $\boldsymbol{M}_{i}^{c}(t)$ is $\int_{t_{0}}^{t} d \tau\left|\boldsymbol{B}_{i}^{c, \text { eff }}(\tau)\right|$, which explicitly involves the past history of $\left|\boldsymbol{B}_{i}^{c, \text { eff }}(t)\right|$. In order to decrease the computation time, we approximate this integral for small time integration steps $d t=t-t_{0} \ll t_{0}$,

$$
\int_{t_{0}}^{t} d \tau\left|\boldsymbol{B}_{i}^{c, \text { eff }}(\tau)\right| \approx\left|\boldsymbol{B}_{i}^{c, \text { eff }}\left(t_{0}\right)\right| d t
$$

In order to assure numerical accuracy of our results for the greatly different experimental parameters studied, we had to make appropriate choices for the numerical parameters used in the calculations, as discussed in the Appendix. Generally, calculations with slow sweep rates require correspondingly small $\alpha / \gamma$ values. For the calculations leading to the results presented in Figs. 1, 2, and $6-9$, we take the numerical parameters $d t=1 \times 10^{-4} \mathrm{~s}$, $B_{\max }=2.0 \mathrm{~T}, N_{t}=1000$, and $N_{B}=500,1000$ and 4000 , respectively, for the different sweep rates studied. For the calculations presented in Figs. 3-5, we take $d t=6 \times 10^{-12}$ $\mathrm{s}, B_{\max }=22.5 \mathrm{mT}, N_{t}=1000$, and $N_{B}=1250$.

\section{RESULTS AND DISCUSSION}

\section{Effects of damping and maximum induction values on the hysteresis}

We first neglect any spin anisotropy effects. In Fig. 1 , we plotted the average over $N_{c}=100$ configurations of the normalized magnetization at the lattice constant $a=1.5 \mathrm{~nm}$, sweep rate $\frac{\Delta B}{\Delta t}=0.005 \mathrm{~T} / \mathrm{s}$, and temperature $T=0.7 \mathrm{~K}$ for the four weak damping rates $\alpha / \gamma=3 \times 10^{-n}$, where $n=10,11,12$, and 13 . These results appear respectively from left to right (right to left) in the upper (lower) part of Fig. 1. The magnetization curves show hysteresis for all four of these $\alpha$ values. For the smallest $\alpha$ value we studied, $\alpha / \gamma=3.0 \times 10^{-13}$, the hysteresis only occurs for external induction magnitudes exceeding $3.0 \mathrm{~T}$, observed by setting $B_{\max }$ above that value, which is well beyond the domain pictured in Fig. 1. We also note that in Fig. 1, the central regions for $\left|\langle M\rangle /\left(N M_{s}\right)\right|<0.8$ are non-hysteretic. For each of these four parameter value choices, the initial curve describing the first increase of the average magnetization from essentially 0 to its saturation value is indistinguishable from subsequent similar curves obtained after completing the full hysteresis paths. Hence, in this case, the main consequence of the choice of $N_{c}=100$ configurations is the

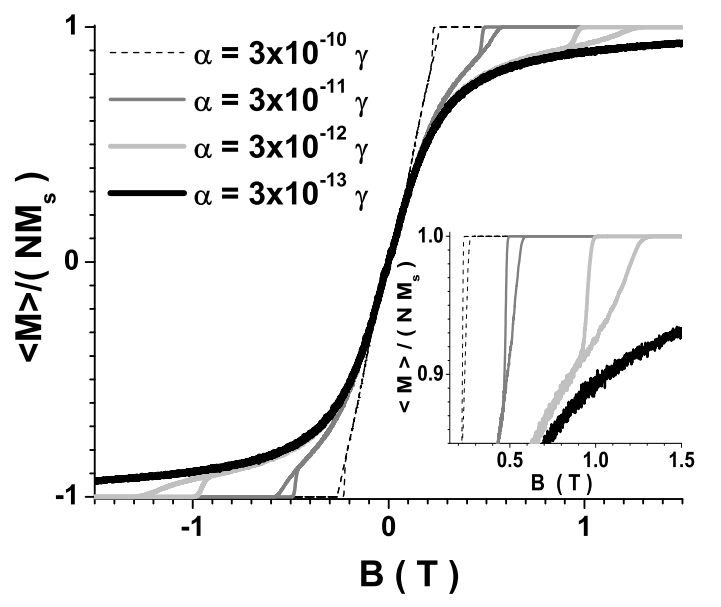

FIG. 1: Magnetization curves for $N_{c}=100, a=1.5 \mathrm{~nm}$, $\frac{\Delta B}{\Delta t}=0.005$ From left to right for $\mathcal{M}>0, \alpha / \gamma=3 \times 10^{-10}$ (dashed), $3 \times 10^{-11}$ (thin dark grey), $3 \times 10^{-12}$ (light grey), $3 \times 10^{-13}$ (thick black). The inset highlights the hysteretic region of the first three of these curves.

improvement in the statistics, reducing the noise that remains most evident in the curves corresponding to the smallest $\alpha$ values.

From the inset to Fig. 1, we see that although the height (in $\langle M\rangle /\left(N M_{s}\right)$ ) of the hysteretic region decreases with decreasing $\alpha$, the width (in $B$ ) of the hysteretic region increases faster with decreasing $\alpha$, so that the overall area of the hysteretic region increases with decreasing $\alpha$. From a computational standpoint, for the parameter values studied in Fig. 1, the smaller the value of $\alpha$, the larger the required value of $B_{\max }$ to produce hysteresis. We also noticed that in these magnetization curves, the hysteresis sets in at the point of an abrupt change in slope in the initial curve, which describes the first increase of the average magnetization from 0 to its saturation value. Moreover, we conclude that $B_{\max }$ must be chosen to guarantee that the system reaches saturation at $B \leq B_{\max }$, because of the different nature of the hysteresis present in each curve. For example, in Fig. 1 the hysteresis can occur only after saturation, but with smaller $a$ values, if the system has not saturated by $B=B_{\max }$, then the magnetization will keep increasing for a number of subsequent $\Delta \boldsymbol{B}$ steps, even though the direction of $\boldsymbol{\Delta} \boldsymbol{B}$ (but not of $\boldsymbol{B}$ ) has been reversed.

\section{Effect of temperature on the hysteresis}

Temperature-independent $\alpha$

We first investigate the role of temperature that arises only from the statistics, Eq. (5), and present our results 


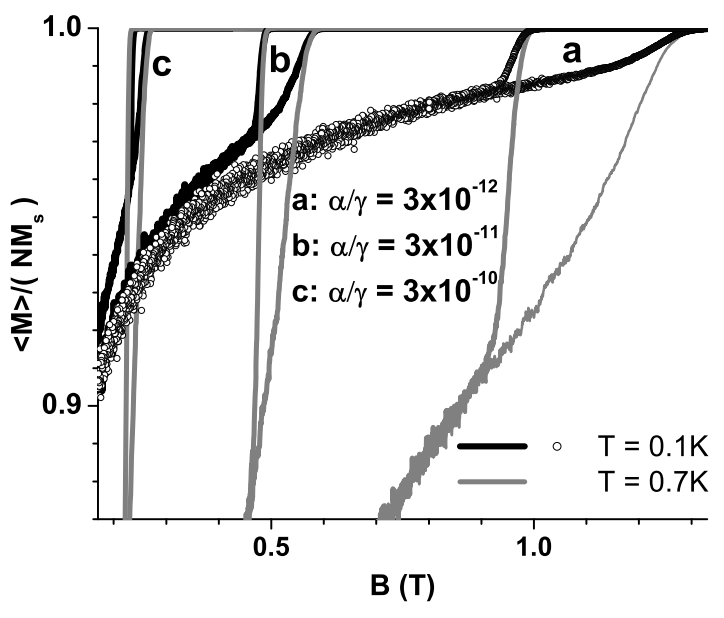

FIG. 2: Shown is the upper hysteretic region of the normalized magnetization curves at $T=0.7 \mathrm{~K}$ (grey) and $T=0.1 \mathrm{~K}$ (black, circles). The $T$-independent damping constants $\alpha / \gamma$ are $3 \times 10^{-12}(\mathrm{a}), 3 \times 10^{-11}(\mathrm{~b})$, and $3 \times 10^{-10}(\mathrm{c})$. The other parameters are the same as in Fig. 1.

for a $T$-independent $\alpha$ in Fig. 2. In this figure, we have replotted the inset of Fig. 1, excluding the curve for $\alpha / \gamma=3 \times 10^{-13}$, for which the hysteresis occurred for $B$ too large to display on the same plot. Otherwise, the parameters are the same as in Fig. 1, except that we have compared our results (grey curves) for $T=0.7 \mathrm{~K}$ shown in Fig. 1 with those (black curves and circles) for $T=0.1 \mathrm{~K}$. Since the evolution of the magnetization with $\boldsymbol{B}$ in this model is independent of $T$, we note from Fig. 2 that the departures of the magnetization curves from the points of saturation are the same at both $T$ values, so that the widths (in $B$ ) of the hysteretic regions are nearly the same. However, the height in $\langle M\rangle /\left(N M_{s}\right)$ of each hysteretic region decreases strongly with decreasing $T$, so that the overall area of each hysteretic region decreases with decreasing $T$. This particular result is in strong contrast to the existing experimental results on SMM's. Nevertheless, our results are reasonable from the point of view of the LLG equation and the way $T$ enters our calculation. We reiterate that we have so far neglected quantum and spin anisotropy effects, the latter of which will be discussed in the following.

We remark that in Fig. 2, $T$ only enters into the equations of motion when the average magnetic moment is evaluated from Eq. (51). As for the Brillouin function that describes the magnetization of a paramagnet in the absence of the dipole interactions, the initial slope of the magnetization at low $B$ increases as $T$ is lowered. This increases the alignment of the moment of each nanomagnet at decreasing $T$, so that the dipole-dipole interactions tend to be maximized, enhancing the effect.
Temperature-dependent $\alpha$

We now consider the effect of the temperature dependence of the damping constant $\alpha$ upon the magnetic hysteresis, focussing upon the case of correspondingly fixed very high sweep and damping rates. We assume that our choice of spin value, $S=5$ for each nanomagnet, satisfies $S \gg 1$. In this limit, Fredkin and Ron showed that the damping of the nuclear spin precession by magnetic dipole coupling to a heat bath, as derived under the assumption of spin-orbit factorization by Wangsness and Bloch, could be readily extended to the spins in magnetic systems. 49, 50] For $S \gg 1$, they found

$$
\alpha(T) / \gamma \approx \frac{T_{0}}{T},
$$

where $T_{0}=2 \hbar \Phi_{11}^{1}\left(1-e^{-\kappa}\right) S^{2} / k_{B} \kappa,[50]$ and $\Phi_{11}^{1}$ is a rate constant (with units of $s^{-1}$ ), the expression for which is a complicated orbital integral arising from the interaction of the local spin with its surrounding molecular electronic orbitals in second-order perturbation theory, [49] and $\kappa=$ $\hbar \gamma B^{\text {eff }} /\left(k_{B} T\right)$. For $\kappa \ll 1, T_{0} \rightarrow 2 \hbar \Phi_{11}^{1} S^{2} / k_{B}$, which can be taken to be independent of $T$ and $B^{\text {eff }}$, so that $\alpha \propto$ $T^{-1}$, but for $\kappa \gg 1, \alpha \propto 1 / B^{\mathrm{eff}}$, which would completely change its effect. Here we only consider the case $\kappa \ll 1$, for which Eq. (9) holds for constant $T_{0}$. We note that, as in Figs. 1 and 2, $T$ also affects the results for the magnetization from the statistics, Eq. (5).

In Fig. 3, we have shown our results, averaged over $N_{c}=100$ configurations, of the normalized magnetization as a function of $B$ in $\mathrm{mT}$, for $a=1.5 \mathrm{~nm}, \frac{\Delta B}{\Delta t}=3000$ $\mathrm{T} / \mathrm{s}, \alpha(T) / \gamma=T_{0} / T, T_{0}=0.3 \mathrm{~K}$, and $T=5 \mathrm{~K}$. For the calculations presented in this figure, we used the numerical parameters $d t=6 \times 10^{-12} \mathrm{~s}, B_{\max }=22.5 \mathrm{mT}$, $N_{t}=1000$, and $N_{B}=1250$. Note that although $a$ has the same value as in Figs. 1 and 2, the sweep and damping $(\alpha(T) / \gamma=0.06)$ rates are six and at least eight orders of magnitude larger than in those figures. For these parameters, there are three regions of hysteresis in the pictured magnetization curve. The left inset is an enlargement of the upper hysteretic region, the mirror image of which occurs in the lower region of the pictured magnetization curve. In contrast to the behavior shown in Figs. 1 and 2 , at the top of the upper hysteretic region, the magnetization does not rise abruptly to its saturation value, but first goes through an extended non-hysteretic region. In addition, there is a central hysteretic region, an enlargement of which is shown in the right inset, along with an enlargement of the same central hysteretic region obtained at $T=0.25 \mathrm{~K}$ with the same set of parameters. We note that at $T=5 \mathrm{~K}$, the onset magnetization averaged over $N_{c}=100$ configurations, pictured by the thin curve in the lower portion of the right inset, does not coincide with the thick curve corresponding to the central hysteresis loop region obtained subsequently to the attainment of the saturation value by the mag- 


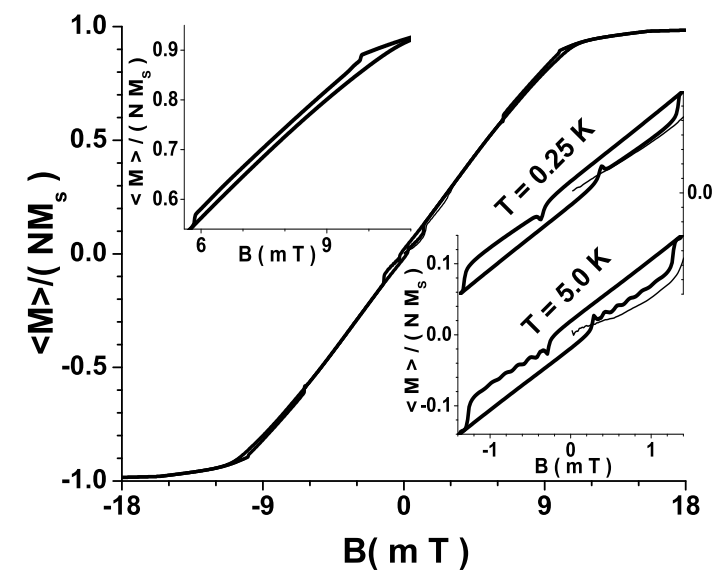

FIG. 3: The magnetization curves for $N_{c}=100$ at $T=5 \mathrm{~K}$, $a=1.5 \mathrm{~nm}$, and $\frac{\Delta B}{\Delta t}=3000 \mathrm{~T} / \mathrm{s}$ with $B_{\max }=22.5 \mathrm{mT}$ and $\alpha(T) / \gamma=T_{0} / T$ for $\hbar g \mu_{B} B^{e f f} /\left(k_{B} T\right) \ll 1$ and $T_{0}=0.3 \mathrm{~K}$ are shown. [50] Left inset: details of the upper portion of the curve. Right inset: details of the central hysteretic portion of the curve shown, along with the central portion of the corresponding curve at $T=0.25 \mathrm{~K}$. The thin curves beginning near to the origin represent the magnetization onsets. These curves are offset for clarity, with the scales on the right (left) sides corresponding to the lower (upper) curves, respectively.

netization. In addition, we note that the thick central hysteresis loop exhibits reproducible oscillations with $B$ independent frequency $f$ at $T=5 \mathrm{~K}$, which oscillations have disappeared at the lower $T=0.25 \mathrm{~K}$ value, for which $\alpha(T) / \gamma=1.2$, pictured in the upper portion of the right inset.

In order to investigate further the differences between the starting magnetization curves and the curves obtained subsequent to saturation, in Fig. 4, we have shown the corresponding central hysteresis loop portion of the magnetization obtained for two individual configurations, using the same experimental and numerical parameters as in Fig. 3, except that $T=10 \mathrm{~K}$, for which $\alpha(T) / \gamma=0.03$. As in Fig. 3, $T$ enters both through the statistical averaging and through the damping, $\alpha(T)$. In Fig. 4, the solid and open circles correspond to the starting magnetizations of the two configurations, and the coincident thick black and thin light grey curves correspond to the central hysteresis loop region of their respective magnetization curves obtained after saturation. Note that after the initial noisy regions, the starting magnetizations for these two configurations exhibit comparably large amplitude oscillations at the frequency $f / 2$, the phases of which are very different. However, after the attainment of the saturation magnetization, these large amplitude oscillations are absent, and replaced by smaller amplitude oscillations at the frequency $f$, which are similar to the oscillations present in our results obtained at

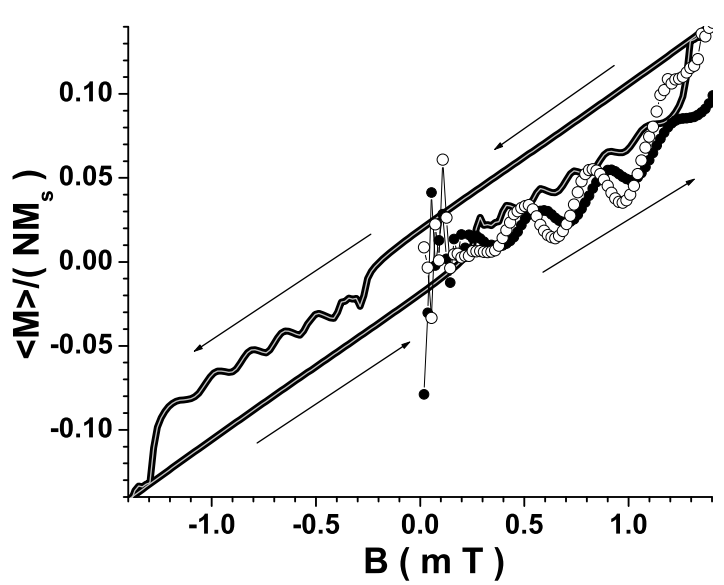

FIG. 4: The central loop and starting magnetization curves for two separate configurations, each with $N_{c}=1$ (open and filled circles) at $T=10 \mathrm{~K}, a=1.5 \mathrm{~nm}$, and $\frac{\Delta B}{\Delta t}=3000 \mathrm{~T} / \mathrm{s}$ with $\alpha(T) / \gamma=T_{0} / T$ for $\hbar g \mu_{B} B^{\text {eff }} / k_{B} T \ll 1$ and $T_{0}=0.3 \mathrm{~K}$ are shown. [50] The thin grey and thick black curves represent the identical behaviors of the central hysteretic loop portion of the magnetization for the same two configurations obtained after saturation. The arrows indicate the direction of the magnetization hysteresis. Here $B_{\max }=22.5 \mathrm{mT}$. See text.

$T=5 \mathrm{~K}$ shown in the lower curves in the right inset of Fig. 3. We note that in the first oscillation present on both sides of the central post-saturation hysteresis loops obtained with these parameters at $T=5$ and $10 \mathrm{~K}$ show additional small amplitude, higher frequency oscillations, which may be higher harmonics of $f$. In addition, the amplitudes of the fifth and sixth oscillations are larger at $10 \mathrm{~K}$ in Fig. 4 than at $5 \mathrm{~K}$ in the lower right inset of Fig. 3.

We remark that the large amplitude oscillations present in the starting magnetizations shown in Fig. 4 are absent in Fig. 3. This occurs due to the randomness of the oscillation phases, which is averaged out in the $N_{c}=100$ configurations studied in Fig. 2 .

From Fig. 4, we therefore conclude that our starting configurations that were chosen to have $|M| / M_{s} \leq 0.1$, appropriate for SMM's, lead to starting magnetization curves that are very different from those that start at the saturation magnetization, but are subsequently identical. That is, after the attainment of saturation, all configurations are identical.

External field directed towards the crystal corners with $\alpha(T)$

We now consider the 3D case of the external magnetic induction directed from the crystal center to one of its corners, $\boldsymbol{B}=B(\hat{\boldsymbol{x}}+\hat{\boldsymbol{y}}) / \sqrt{2}$, the (110) direction. In Fig. 5, we show the resulting central hysteresis region obtained 


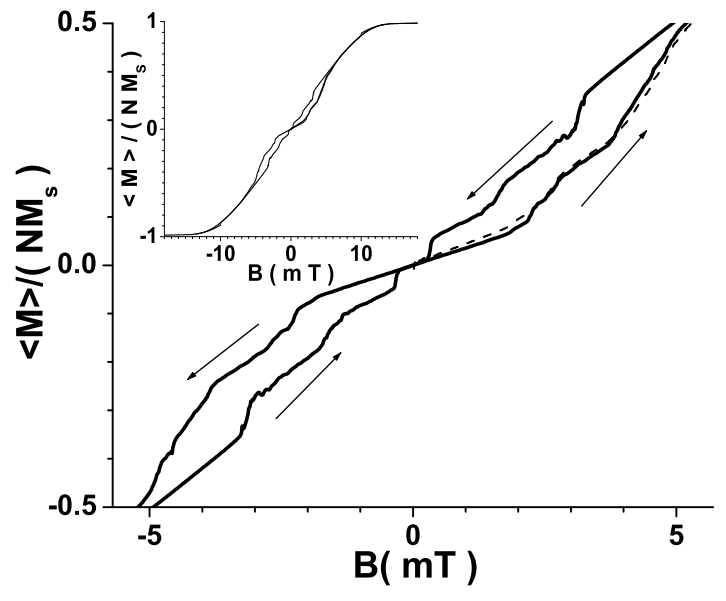

FIG. 5: The central loops (solid curves) of the magnetization curve for $N_{c}=50, N=5 \times 5 \times 4=100$, with $\boldsymbol{B}$ along the [110] direction $[\boldsymbol{B} \|(\hat{\mathbf{x}}+\hat{\mathbf{y}}) / \sqrt{2}]$ at $T=10 \mathrm{~K}$, with $a=1.5 \mathrm{~nm}$, $B_{\max }=22.5 \mathrm{mT}$, and $\frac{\Delta B}{\Delta t}=3000 \mathrm{~T} / \mathrm{s}$ with $\alpha(T) / \gamma=T_{0} / T$, and $T_{0}=0.3 \mathrm{~K}$. The dashed curve is the starting magnetization curve. The arrows indicate the direction of the hysteresis. Inset: the full magnetization curve. See text.

from our calculations for $N_{c}=50, N=5 \times 5 \times 4, T=10$ $\mathrm{K}, a=1.5 \mathrm{~nm}$, and $\frac{\Delta B}{\Delta t}=3000 \mathrm{~T} / \mathrm{s}$ with $\alpha(T) / \gamma=$ 0.03. In this case, it is sufficient to set $B_{\max }=22.5$ $\mathrm{mT}$, which leads to full saturation. We note that for this field direction, a small $(-6 \mathrm{mT}<B<6 \mathrm{mT})$ hysteresis region appears on either side of the origin, which is rather central to the full magnetization curve, but vanishes over a small region close to the origin. There are also tiny hysteresis regions near to saturation that appear as dots in the inset depicting the full magnetization curve.

The nearly central hysteretic regions shown in Fig. 5 exhibit reproducible jumps at specific $B$ values, similar to those observed at low $T$ in SMM's. However, we note that in this figure, we have taken $T=10 \mathrm{~K}$, and have used a classical spin model. We also note that we have used a rather small sample $(N=100)$ with a fast sweep rate and a large damping coefficient in our calculations, and caution that such behavior might not be present in large single crystals, especially with much slower sweep rates. Nevertheless, this figure demonstrates that steps in the magnetization do not necessarily have a quantum origin, and that the sample shape can lead to unusual hysteresis effects.

\section{Effect of sweep rate on the hysteresis}

From the curves obtained using the same numerical parameters as in Fig. 1 for different induction sweep rates at a fixed, small damping rate in Fig. 6, it is clear

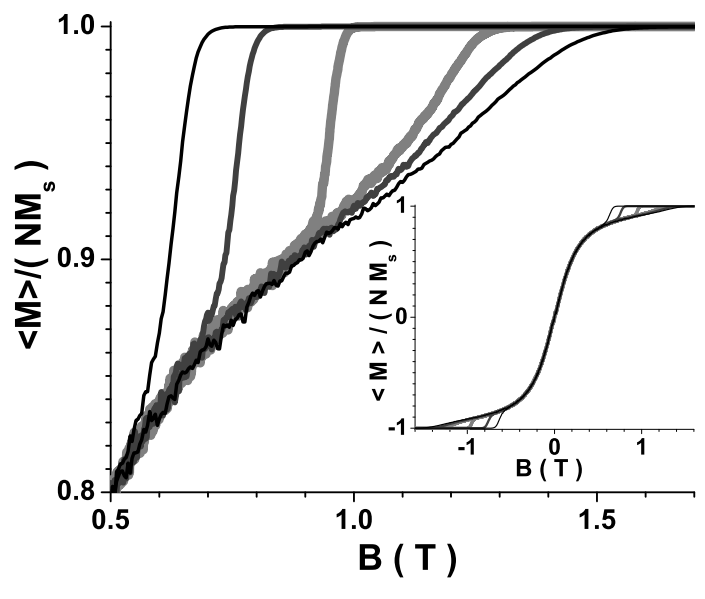

FIG. 6: Hysteretic region of $\mathcal{M}(B)$ at $0.7 \mathrm{~K}, \alpha / \gamma=3 \times 10^{-12}$, and $a=1.5 \mathrm{~nm}$, for the sweep rates $\frac{\Delta B}{\Delta t}=0.04 \mathrm{~T} / \mathrm{s}$ (thin black), $0.02 \mathrm{~T} / \mathrm{s}$ (dark grey), and $0.005 \mathrm{~T} / \mathrm{s}$ (thick light grey). The inset shows the entire curves.

that stronger hysteresis is found for higher sweep rates, in agreement with experiments on a variety of nanomagnets, including SMM's. This shows that the reversibility of the process depends on how close to equilibrium the sweep rate allows the nanomagnet spins to reach. That is, although for different sweep rates the external induction is increased by the same amount $\Delta \boldsymbol{B}$, at higher sweep rates, the time $\Delta t$ allowed for the nanomagnets to evolve towards equilibrium is less. This makes the process less reversible and the hysteresis loops larger.

We also note that at the much higher sweep and damping rates studied in Figs. 3, 4, the magnetization also exhibits a central hysteretic region, which exhibits oscillations at $T$ values not too low and/or damping constants not too large.

\section{Effect of lattice constant on the hysteresis}

In Fig. 7, we show hysteresis curves for two different values of the lattice constant $a$, obtained using the same numerical parameters as in Fig. 1. For weaker dipole-dipole interactions (larger $a$ ), the rise in the magnetization is steeper with increasing $\boldsymbol{B}$, and the rapid decrease in the magnetization from its saturation value upon decreasing $\boldsymbol{B}$ occurs at a smaller value of $|\boldsymbol{B}|$. Furthermore, we shall see that dipolar interactions do not promote hysteresis in these systems, but suppress it. Actually, the same conclusion was found recently for magnetic nanoparticles in the framework of the generalized mean-field approximation. 55 .

This peculiar hysteresis is easily understood by analyzing the LLG equation. If the nanomagnet magnetiza- 


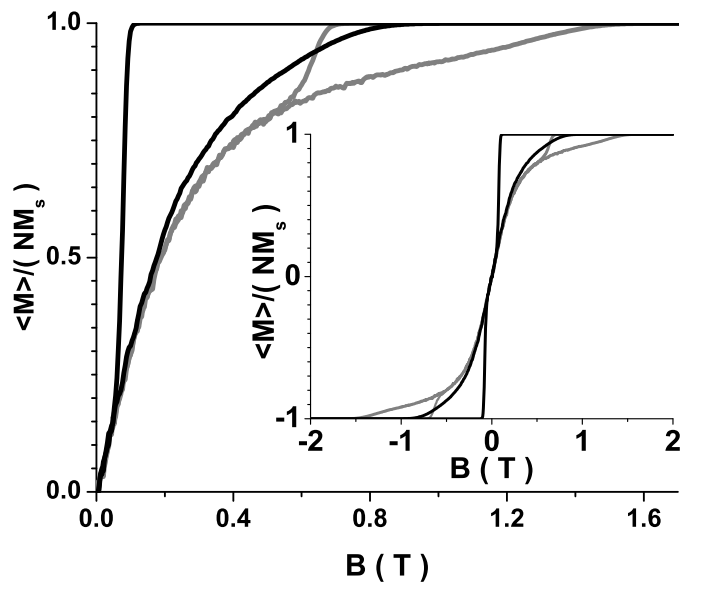

FIG. 7: Magnetization curves for lattice constants $a=1.5$ $\mathrm{nm}$ (grey) and $a=2.5 \mathrm{~nm}$ (black). $\frac{\Delta B}{\Delta t}=0.04 \mathrm{~T} / \mathrm{s}, \alpha=$ $3 \times 10^{-12} \gamma, T=0.7 \mathrm{~K}$.

tion $\boldsymbol{M}_{i}^{c}$ is parallel to its local magnetic induction $\boldsymbol{B}_{i}^{c, \text { eff }}$, $d \boldsymbol{M}_{i}^{c} / d t=0$, as it will remain thereafter, so that $\boldsymbol{M}_{i}^{c}$ has reached equilibrium. The only chance the system has to decrease its magnetization from its saturation value is through the combined weak dipolar induction, which strengthens with decreasing lattice parameter $a$. The dipolar induction can oppose the system from remaining completely magnetized, since it has small, but nonvanishing components. Therefore, even when $\boldsymbol{B}$ reaches its maximum (finite) amplitude $B_{\max }$ and the misalignments of each $\boldsymbol{M}_{i}^{c}$ with $\boldsymbol{B}$ are negligible, dynamic equilibrium will not generally have been attained due to the limited time allowed for relaxation before the next change in $\boldsymbol{B}$. There will remain a slight deviation between the directions of the $\boldsymbol{B}_{i}^{c, \text { eff }}$ and the $\boldsymbol{M}_{i}^{c}$ due to the presence of the $\boldsymbol{B}_{i}^{c, \text { dip }}$, which is especially important when $B$ decreases from $B_{\max }$.

Of course, it is harder to decrease $\mathcal{M}$ at the very beginning of the induction cycle. This is precisely the cause of the hysteretic behavior, given that changes in $\boldsymbol{M}_{i}^{c}$ are proportional to $\left|\boldsymbol{M}_{i}^{c} \times \boldsymbol{B}_{i}^{c, \text { eff }}\right|$, which nearly vanishes when the direction of the incremental induction has just been reversed. We conclude then that the smaller the lattice parameter (the stronger the dipolar induction), the greater the deviation of $\boldsymbol{M}_{i}^{c}$ from the direction of $\boldsymbol{B}_{i}^{\text {eff }}$. Hence, the easier it is to decrease $\mathcal{M}$, making the magnetization curve less hysteretic. This is shown in Fig. 7, in which the magnetization curves resemble those obtained for $\mathrm{Mn}_{4}$ SMM's. 31] Those data show an abrupt decrease in $\mathcal{M}$ at nearly zero external induction that is not evident in the magnetization curves of other SMM's. 28]

It is important to note that the curves in Figs. 1-7 do not show the strong hysteresis observed experimentally in most SMM's, which is especially large in the central region of the $\mathcal{M}(B)$ curves. We remind the reader of our intent to focus upon the effects of the dipole-dipole interactions, whereas the most important features of SMM's involved in their low- $T$ relaxation of the magnetization are generally thought to be their quantum structure and magnetic anisotropy. Nevertheless, for this entirely classical and magnetically isotropic system, we are indeed finding hysteretic curves. In addition, the sweep rates in Figs. 1, 2, 6, and 7 are comparable to those used in experimental SMM studies. At much larger sweep rates, such as were studied in Figs. 3-5, an hysteretic central region was found. However, the sizes and $T$ dependencies of these hysteretic regions were still respectively much smaller and qualitatively different than observed in SMM's.

\section{Effect of spin anisotropy upon the hysteresis}

It is straightforward to generalize our model to include some of the effects of magnetic spin anisotropy. Here we assume the nanomagnets contain sufficiently many spins that their quantum nature can be neglected. We note that SMM's at low $T$ values behave as quantum entities, because of the small number of spins in each nanomagnet. In those systems, most workers have assumed the in addition to the isotropic Heisenberg and Zeeman interactions, the magnetic anisotropy terms could also be written in terms of components of the global spin operator $\boldsymbol{S}$, with the overall dominant terms often written as $-D S_{z}^{2}-E\left(S_{x}^{2}-S_{y}^{2}\right)$. [56] However, portions sufficiently large for model comparison of the low- $T$ magnetization curves of two $\mathrm{Fe}_{2} \mathrm{SMM}$ dimers have been studied experimentally. 57, 58] In neither antiferromagnetic dimer case was any evidence for either of those types of spin anisotropy present. [59 In contrast, in one of those cases, strong evidence for a substantial amount of local, single-ion spin anisotropy, in which the individual spins within a dimer align relative to the dimer axis, is present in the data. 57, 59] In addition, the global anisotropy in the ferromagnetic $\mathrm{SMM} \mathrm{Mn}_{6}$ is extremely weak. [60] Since the precise quantum nature of more complicated SMM's appears therefore to be poorly understood, we shall investigate the quantum features of the magnetic hysteresis curves in SMM's, including some effects of local spin anisotropy, in a subsequent publication. 54

We therefore restrict our investigations of the role of magnetic anisotropy upon the magnetization curves of arrays of nanomagnets to the simplest classical model of spin anisotropy,

$$
\boldsymbol{B}_{i}^{c, \text { eff }}=\boldsymbol{B}+\boldsymbol{B}_{i, \text { dip }}^{c}+\mu_{0} \boldsymbol{H}_{A},
$$

where we take $\boldsymbol{B}=B \hat{\boldsymbol{x}}$ and studied the cases $\boldsymbol{H}_{A}=H_{A} \hat{\boldsymbol{x}}$ and $\boldsymbol{H}_{A}=H_{A} \hat{\boldsymbol{z}}$. This is the 3D analogue of the model studied by KS. 45] In this model, the magnetic anisotropy 


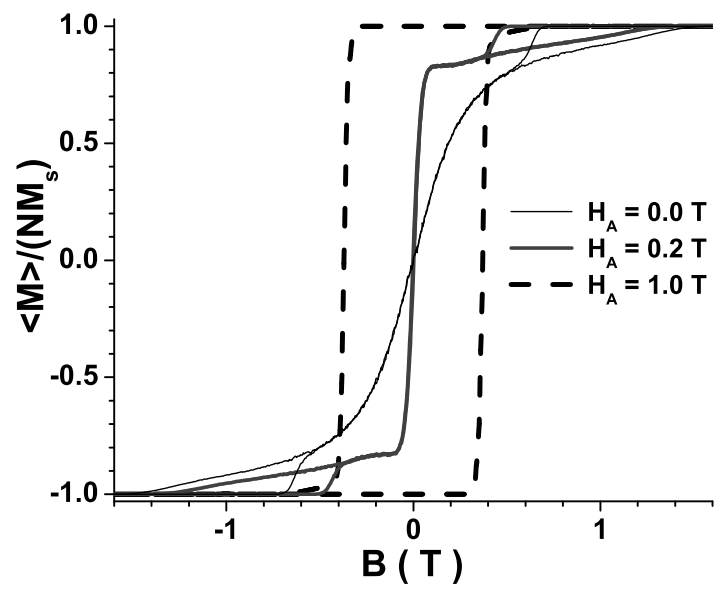

FIG. 8: Parallel 3D magnetization curves including different anisotropy field $\boldsymbol{H}_{A}=H_{A} \hat{\boldsymbol{x}}$ strengths, with the external induction $\boldsymbol{B} \| \boldsymbol{H}_{A}, \quad \mu_{0} H_{A}=0$ (thin black), $0.2 \mathrm{~T}$ (dark grey), and $1.0 \mathrm{~T}$ (thick dashed), respectively. For each curve, $N_{c}=100, N=5 \times 5 \times 4=100, \alpha / \gamma=3 \times 10^{-12}, a=1.5$ $\mathrm{nm}, \frac{\Delta B}{\Delta t}=0.04 \mathrm{~T} / \mathrm{s}, T=0.7 \mathrm{~K}$, and $B_{\max }=2.0 \mathrm{~T}$.

of each of the nanomagnets points in the same direction, and in our finite sized crystal consisting of $5 \times 5 \times 4$ nanomagnets on a cubic lattice, our chosen direction is one of the most general ones. We first performed two studies of the magnetic hysteresis in this model, for which the anisotropy field $\boldsymbol{H}_{A}$ is directed respectively along (100), $\| \boldsymbol{B}$, and (001), $\perp \boldsymbol{B}$, and our results are shown in Figs. 8-9, respectively. For both anisotropy field directions, we take $N_{c}=100, N=5 \times 5 \times 4=100$, $\alpha / \gamma=3 \times 10^{-12}, a=1.5 \mathrm{~nm}, \frac{\Delta B}{\Delta t}=0.04 \mathrm{~T} / \mathrm{s}, T=0.7$ $\mathrm{K}$, and $B_{\max }=2.0 \mathrm{~T}$. The sweep rates used in Figs. 8-9 are slightly faster than those used in SMM experiments but much slower than those used in the calculations of KS. Since $a=1.5 \mathrm{~nm}$ in these curves, these curves also represent the strongest realistic dipolar interaction we studied.

In Fig. 8, we show the portions of the parallel magnetization curves with $\boldsymbol{B}\left\|\boldsymbol{H}_{A}\right\| \hat{\boldsymbol{x}}$, that exhibit the resulting regions of magnetic hysteresis for three $H_{A}$ values. For the $\mu_{0} H_{A}=0,0.2$, and $1.0 \mathrm{~T}$ values shown, all three curves are hysteretic, but the two lower $H_{A}$ values do not lead to a central hysteresis region. Nevertheless, the largest anisotropy value, $H_{A}=1.0 \mathrm{~T}$, leads to a strong central hysteresis. We remark that the trends shown in Fig. 7 are rather different from those obtained for a single magnetic particle with magnetic anisotropy. 5

In Fig. 9, we show the portions of the 3D perpendicular magnetization curves exhibiting the resulting regions of magnetic hysteresis for the five anisotropy fields $\mu_{0} H_{A}=0,1 \mathrm{mT}, 12 \mathrm{mT}, 0.5 \mathrm{~T}$, and $1.0 \mathrm{~T}$, with the magnetic induction $\boldsymbol{B}\left\|\hat{\boldsymbol{x}} \perp \boldsymbol{H}_{A}\right\| \hat{\boldsymbol{z}}$. In each case, hys-

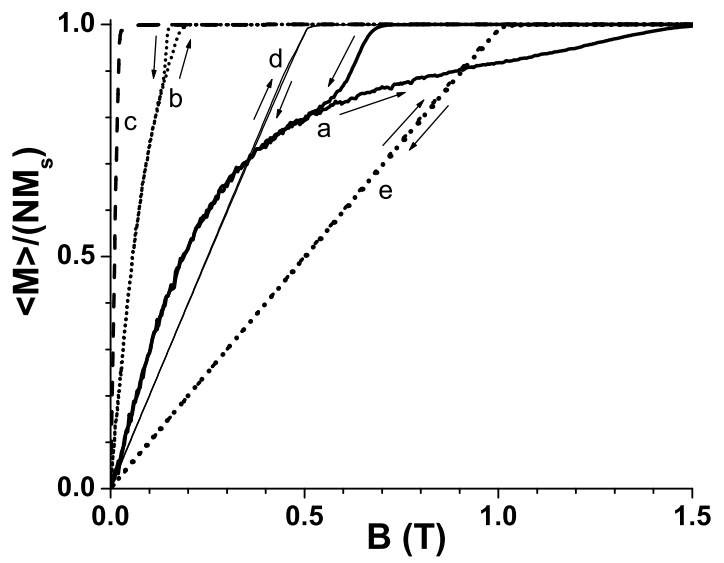

FIG. 9: Upper region of the 3D perpendicular magnetization curves with the external induction $\boldsymbol{B}=B \hat{\boldsymbol{x}} \perp \boldsymbol{H}_{A}=H_{A} \hat{\boldsymbol{z}}$, for different values of $H_{A}$. Curves (a)-(e) correspond to $\mu_{0} H_{A}=0,1 \times 10^{-3}, 1.2 \times 10^{-2}, 0.5,1.0 \mathrm{~T}$, respectively. The other parameters are the same as in Fig. 7. The arrows indicate the directions of the field sweeps.

teresis occurs near to magnetic saturation, but is absent in the central region for small magnetic induction. At $\mu_{0} H_{A}=1.0 \mathrm{~T}$, this is distinctly different from the large central hysteretic region observed for parallel anisotropy. Note that the slope $d \mathcal{M} / d B$ at small $B$ is non-monotonic with increasing $H_{A}$, as it has a minimum at curve (c), corresponding to $\mu_{0} H_{A}=12 \mathrm{mT}$.

Thus, we conclude that it is possible to obtain a central hysteresis region using this classical model of dipolar interactions with constant spin anisotropy. However, our results suggest that such central hysteresis regions only arise for the magnetic induction parallel to the spin anisotropy direction, and for sufficiently strong anisotropy fields, $H_{A} \geq H_{A}^{\min }$, where $1.0 \mathrm{~T}>\mu_{0} H_{A}^{\min }>$ $0.2 \mathrm{~T}$.

\section{DIPOLAR INTERACTION, INDUCTION SWEEP RATE, AND ANISOTROPY DEPENDENCIES FOR A 2D SYSTEM}

To estimate the importance of the dipolar induction (especially when it becomes comparable to the external induction), the anisotropy and the sweep rate, we have reproduced one of the $2 \mathrm{D}$ calculations of KS. 45] The KS calculation we chose to reproduce was pictured in their Fig. 2(i), and is shown here as the left panel of Fig. 10. Then, we changed some experimental parameters to see how the results depend on the anisotropy strength, sweep rate, and lattice parameter.

Our calculations for a cubic lattice consisting of four 25-particle layers differ from those of KS in many 
ways. 45. They used a $2 \mathrm{D}$ square lattices of cylindrical nanodots (here, we take their $5 \times 5$ lattice with external induction aligned along an array's diagonal), included a shape-dependent anisotropy field perpendicular to the lattice, performed their calculations at $T=0$, used a much larger damping constant than we generally did it for 3D systems, and did not average their results over an ensemble of 2D samples, because such systems do not show variations in the resulting hysteresis loops for different initial states. Nevertheless, we both integrated the LLG equation using the Runge-Kutta algorithm, and surprisingly, KS's system turned out to be very sensitive to the dipolar field strength. The effective induction they considered can be written as

$$
\boldsymbol{B}_{i}^{c, \text { eff }}=\boldsymbol{B}+\boldsymbol{B}_{i, \mathrm{dip}}^{c}+\mu_{0} H_{A} \hat{\boldsymbol{z}} .
$$

For lattice constant $a=1.5 \mathrm{~nm}$, spin $S=5$, and $V / a^{3}=0.5$, where $V$ is the volume of the nanomagnet, the saturation magnetization is $M_{s} \approx 55$ Oe. Then, they took the dimensionless $d t=5 \times 10^{-3}$, which implies a real time interval $d t=5.17 \times 10^{-12} \mathrm{~s}$. If the system evolves during 700 time steps $d t$ for some fixed value of $\boldsymbol{B}$, then $\boldsymbol{B}$ is changed every $\Delta t \approx 3.62 \times 10^{-9}$ s. On the other hand, KS chose a maximum external induction $B_{\max }=2 \mu_{0} M_{s} \approx 1.1 \times 10^{-2}$ T. In addition, they took fixed induction steps of magnitude $\Delta B=2 \times 10^{-3} \mu_{0} M_{s} \approx 1.1 \times 10^{-5} \mathrm{~T}$. Therefore, we estimate their resulting sweep rate to be $\frac{\Delta B}{\Delta t} \approx 3 \times 10^{3}$ $\mathrm{T} / \mathrm{s}$, as in our $3 \mathrm{D}$ results shown in Figs. 3,4 .

In the absence of any specific information, we then had to induce the value of the anisotropy field that KS used to obtain their figure. Fortunately, as discussed in the following, the results are rather insensitive to it, unless $H_{A}$ becomes comparable to $B_{\max } / \mu_{0}$. In the right panel of Fig. 10, our 2D calculation with $\mu_{0} H_{A}=0.75 \mathrm{mT}$ are shown, and by comparing that figure with Fig. 2(i) of KS pictured in the left panel of Fig. 10, we see that the agreement is remarkably good.

Very recently, Takagaki and Ploog (TP) used a fourthorder Runge-Kutta procedure to integrate the LLG equations with $N \times N 2$ D nanomagnet lattices with magnetic anisotropy and dipole-dipole interactions. 61 They used a fixed time interval $d t=0.1 \hbar /\left(\gamma M_{s}\right), 20$ times as fast as that used by KS, 45] and continued interating until no further changes in the nanomagnet spin configurations were obtained. They obtained results for $N=5$ which they described as considerably different from those of KS, with a somewhat different magnetization loop and a larger area of the hysteretic regions. 61] Although they claimed that their fourth-order procedure was intrinsically more accurate than the second-order one used by $\mathrm{KS}$ and by us, the fact that we obtained the excellent agreement pictured in Fig. 10 with one of the $N=5$ results of KS suggests that the procedure used by TP might have been less accurate than they claimed. 61.
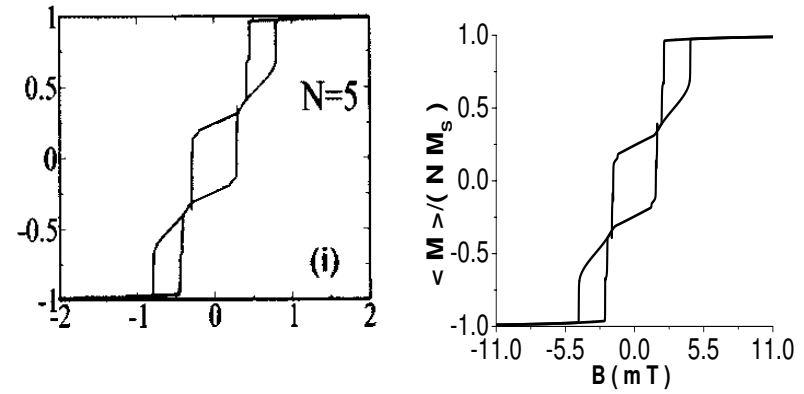

FIG. 10: (left) Hysteresis loop $\mathcal{M}(B)$ in units of $M_{s}$, for a weakly coupled array of $5 \times 5$ ferromagnetic nanodots in a square lattice on the $x y$ plane, from Fig. 2(i) of KS. The external induction is applied along the array diagonal $\left(45^{\circ}\right.$ from the $x$ axis). [45]. (right) Our results calculated for $N_{c}=1$ with $5 \times 5$ nanomagnets on a square lattice, $\alpha / \gamma=0.6, T=$ $0 \mathrm{~K}, \frac{\Delta B}{\Delta t}=3000 \mathrm{~T} / \mathrm{s}, \mu_{0} H_{A}=7.5 \times 10^{-4} \mathrm{~T}, \boldsymbol{B}=B(\hat{\boldsymbol{x}}+\hat{\boldsymbol{y}}) / \sqrt{2}$.

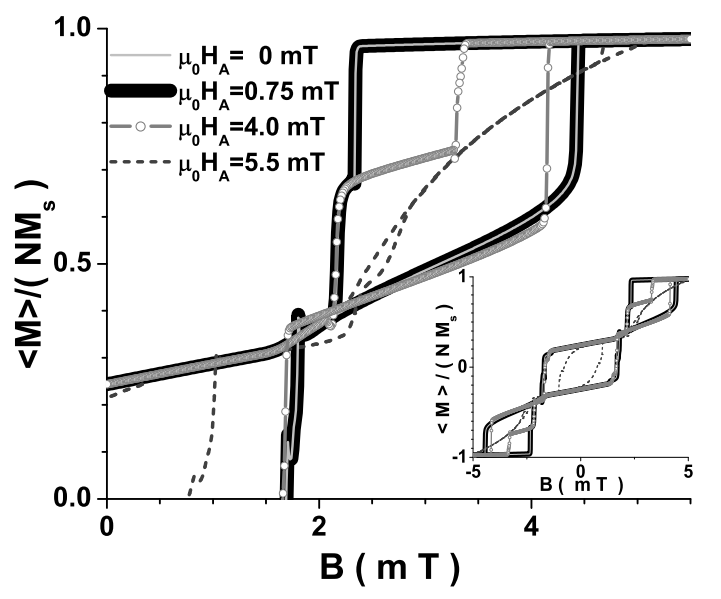

FIG. 11: Hysteresis loops for different strengths of $H_{A}$ for $5 \times 5$ nanomagnets on a square lattice with $N_{c}=1 . S=5$, $T=0 \mathrm{~K}, a=1.5 \mathrm{~nm}, \frac{\Delta B}{\Delta t}=3000 \mathrm{~T} / \mathrm{s}, \alpha / \gamma=0.6, \boldsymbol{B}=B(\hat{\boldsymbol{x}}+$ $\hat{\boldsymbol{y}}) / \sqrt{2}$. The thin grey and thick black curves with $\mu_{0} H_{A}=$ $0,0.75 \mathrm{mT}$, respectively, are nearly indistinguishable. The small grey circles and dashed curves correspond to $\mu_{0} H_{A}=$ 4.0,5.5 $\mathrm{mT}$, respectively. The inset shows the entire curves, which are symmetric with respect to the origin.

\section{Anisotropy field dependence of the hysteresis}

We first investigated the effects of changing the strength of the anisotropy fields, and presented our results in Fig. 11. The most important issue about the results shown in Fig. 11 is the fact that the curve obtained by KS (the left panel of Fig. 10) is basically independent of the anisotropy field $H_{A}$ for sufficiently small $H_{A}$. That is, there are no essential differences between 
that curve reproduced in the right panel of Fig. 10 with $\mu_{0} H_{A}=7.5 \mathrm{mT}$, and the one with $H_{A}=0$. Strong deviations from these essentially identical curves appear for $\mu_{0} H_{A} \geq 4 \mathrm{mT}$, however. Since identical behavior is obtained without any anisotropy, this implies that all hysteretic features (including the stepped magnetization and demagnetization) are due to the dipolar interaction. $H_{A}$ becomes important only when it is comparable to $B_{\max } / \mu_{0}$ and tends to close the hysteresis loops, starting from the lower and upper loops.

We note that by comparing Fig. 11 with Fig. 9, the details of the hysteresis obtained with $H_{A}=0$ for $\boldsymbol{B}$ along the (110) direction are different in 3D and 2D samples. The hysteresis is much larger in the 2D case pictured in Fig. 11, and has a large loop in the central region that does not vanish at the origin, plus large loops that extend up to saturation. In the $3 \mathrm{D}$ case constructed from four 2D planes each equivalent to that used in the calculation shown in Fig. 11, the magnitude of the hysteresis is reduced and its details have been greatly altered.

\section{Induction sweep rate dependence of the hysteresis}

In Fig. 12, we show our results for a single configuration of a square $2 \mathrm{D}$ lattice with $N=5 \times 5$ for different sweep rates, keeping the other parameters fixed at $\mu_{0} H_{A}=0.75 \mathrm{mT}, \alpha / \gamma=0.6, a=1.5 \mathrm{~nm}, S=5, T=0$, and $\boldsymbol{B}=B(\hat{\boldsymbol{x}}+\hat{\boldsymbol{y}}) / \sqrt{2}$. From Fig. 12, we note that the hysteresis is nearly independent of induction sweep rate over the range 300 to $6000 \mathrm{~T} / \mathrm{s}$, distinctly different from the strong dependence found in 3D systems shown in Fig. 5.

\section{Lattice parameter dependence of the hysteresis}

In Fig. 13, we have illustrated the effect of the lattice constant $a$ upon the hysteresis. In this figure, we kept the other parameters fixed at $S=5, T=0, \frac{\Delta B}{\Delta t}=3000 \mathrm{~T} / \mathrm{s}$, $\mu_{0} H_{A}=0.75 \mathrm{mT}, \alpha / \gamma=0.6$, and $\boldsymbol{B}=B(\hat{\boldsymbol{x}}+\hat{\boldsymbol{y}}) / \sqrt{2}$. As $a$ is varied from 2.0 to $1.25 \mathrm{~nm}$, the upper portions of the hysteresis curves appear from left to right, respectively. From Fig. 13, it is readily seen that the magnetization curves are very sensitive to $a$ and hence to the strength of the dipolar interaction, which is proportional to $a^{-3}$. Our results for $a=2.5 \mathrm{~nm}$ exhibit a smaller hysteresis shifted further to the left, and all indications of steps have disappeared. Although not shown in Fig. 13, as $a$ is increased further to $3.0 \mathrm{~nm}$, the hysteresis almost disappears entirely. We deduce that stronger dipolar interactions (smaller $a$ ) result in larger hysteresis loops containing increased widths of additional steps.

We then infer that contrary to the conclusion found for the 3D systems (based upon much smaller damping

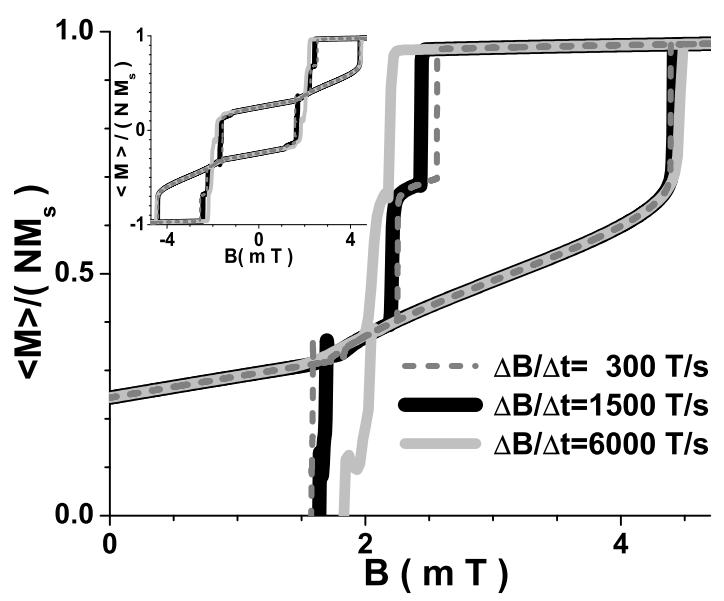

FIG. 12: Hysteresis loops for different induction sweep rates with $5 \times 5$ nanomagnets on a square lattice with $N_{c}=1$. $S=5, T=0 \mathrm{~K}, a=1.5 \mathrm{~nm}, \mu_{0} H_{A}=0.75 \mathrm{mT}, \alpha / \gamma=$ 0.6. The dashed grey, thick black, and light solid grey curves correspond to $\frac{\Delta B}{\Delta t}=300,1500,6000 \mathrm{~T} / \mathrm{s}$, respectively. The inset shows the entire curves. $\boldsymbol{B}=B(\hat{\boldsymbol{x}}+\hat{\boldsymbol{y}}) / \sqrt{2}$.

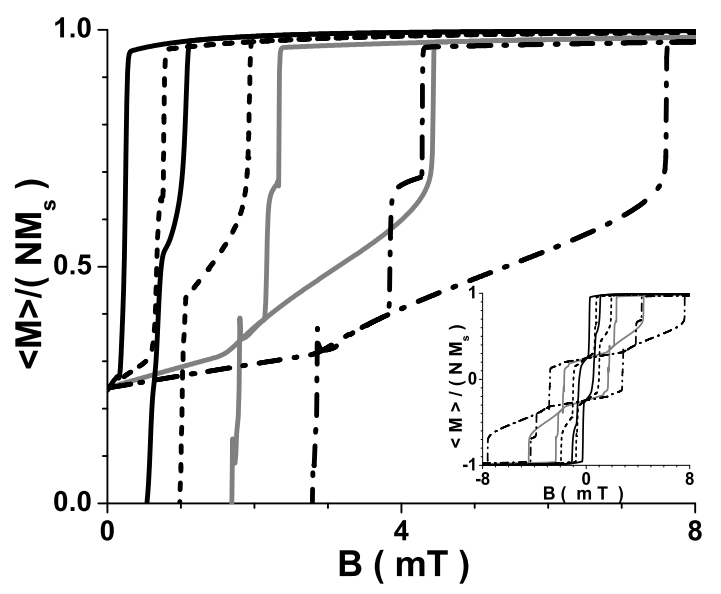

FIG. 13: Hysteresis loops for lattice parameters $a=2.5 \mathrm{~nm}$ (solid black), $a=2.0 \mathrm{~nm}$ (dashed black), $a=1.5 \mathrm{~nm}$ (solid grey), and $a=1.25 \mathrm{~nm}$ (dot-dashed black), for $5 \times 5$ nanomagnets on a square lattice with $N_{c}=1 . S=5, T=0 \mathrm{~K}$, $\frac{\Delta B}{\Delta t}=3000 \mathrm{~T} / \mathrm{s}, \mu_{0} H_{A}=7.5 \times 10^{-4} \mathrm{~T}, \alpha / \gamma=0.6$. The inset shows the entire curves. $\boldsymbol{B}=B(\hat{\boldsymbol{x}}+\hat{\boldsymbol{y}}) / \sqrt{2}$.

coefficients and much slower sweep rates), the dipolar interactions promote a hysteretic behavior in this $2 \mathrm{D}$ system. 


\section{SUMMARY AND CONCLUSIONS}

We first found $N_{c}=100$ sample configurations with an overall magnetization close to 0 . We then solved the Landau-Lifshitz-Gilbert equation for a 3D cubic lattice of $N=5 \times 5 \times 4$ nanomagnets, subject to dipole-dipole interactions and spin anisotropy. These results should be relevant for an array of Stoner-Wolfarth nanomagnets, and to some extent, single molecule magnets, although the quantum nature of the latter has so far been neglected. In the absence of spin anisotropy, we varied the magnetic induction sweep rate $\frac{\Delta B}{\Delta t}$, the damping constant $\alpha$, the lattice constant $a$, and the temperature $T$. We also considered the effects of a $T$-dependent damping constant of the form $\alpha(T) / \gamma=T_{0} / T$ suggested by Fredkin and Ron. For slow sweep rates and small $\alpha$ relevant for experimental studies on single molecule magnets, magnetic hysteresis appears in the regions of the magnetization curves near to saturation, the area and onset magnetic induction strength of which increases with decreasing $\alpha$ and increasing sweep rate. With decreasing $T$, the onset magnetization magnitude of the hysteretic regions near to saturation decreases. With decreasing a corresponding to increased dipole-dipole interaction strengths, the onset of the hysteresis regions near to saturation appears at increasing magnetic induction magnitude.

At much larger sweep rates and damping constants, the magnetization curves attain saturation at much smaller applied magnetic induction strengths. The hysteretic regions just below saturation have moved somewhat below saturation, and a new central hysteretic region appears. As one follows the magnetization curve for a single configuration, the starting curve exhibits oscillations at a rather constant (magnetic induction independent) frequency $f / 2$, but the phase of the magnetization oscillations is a random function of the configuration. After the attainment of magnetic saturation, this central hysteretic region exhibits oscillations at $f$, twice that frequency, possibly with weak higher harmonics, for $T$ not too low, which are independent of the configuration.

When the applied magnetic induction is in the (110) direction (from the sample center to one of its corners), magnetic hysteresis exhibiting steps and jumps appears within the central region, but vanishes at and very near to the origin. Although these step-like features are suggestive of the behavior seen in single molecule magnets, they are present at rather high $T$ values, as they arise from the classical sample shape effects.

In the presence of the magnetic anisotropy field $\boldsymbol{H}_{A}$, an applied magnetic induction parallel to the anisotropy axis leads to a large central hysteresis region, provided that the magnitude of the spin anisotropy is sufficiently large. For the applied magnetic induction perpendicular to the magnetic anisotropy, no central hysteresis region is present, although a small amount of hysteresis near to saturation persists for sufficiently small spin anisotropy strength, and the slope of the magnetization curve at the origin is non-monotonic, exhibiting a maximum at a particular small value of the spin anisotropy strength. These effects for the spin anisotropy are qualitatively in agreement with those in many types of arrays of nanomagnets, including single molecule magnets.

As a test of our numerical procedure, we studied the simplified $5 \times 52 \mathrm{D}$ square lattice with a perpendicular spin anisotropy field $\boldsymbol{H}_{A}$ using the same procedure, and for a particular set of parameters, obtained quantitative agreement with a hysteresis curve obtained for that system by Kayali and Saslow. 45. We showed that their hysteresis curve is basically independent of $H_{A}$ until $\mu_{0} H_{A}$ is on the order of the external induction. We also demonstrated that the magnetic hysteresis does not depend significantly upon the magnetic induction sweep rate, as opposed to the dependence we found in our 3D system. In addition, we found that the magnetization of the $2 \mathrm{D}$ system is very sensitive to variations in the lattice parameter $a$. Finally, we noticed that although dipolar interactions also oppose the magnetization process in 2D systems, increasing the onset magnetic induction strength for the attainment of saturation as in 3D systems, they increase the area of the hysteresis, a behavior opposite to that found for the 3D system with a much smaller damping coefficient and much slower sweep rate.

We expect our results to be relevant to the magnetization processes in a variety of nanomagnet arrays, especially those approximating arrays of Stoner-Wolfarth particles. In addition, some of the features we obtained should be relevant to single molecule magnets, although the temperature dependence of the effects is not in agreement with experiments on those materials. Further studies of the magnetic hysteresis using quantum models of the nanomagnets and their various anisotropy types is warranted, and will be addressed subsequently. [54]

\section{ACKNOWLEDGMENTS}

This work was supported in part by the NSF under grant number NER-0304665.

\section{APPENDIX A}

We rotate our reference frame at every integration time step in such a way that $\boldsymbol{B}_{i}^{c, \text { eff }}$ is along the $z$ axis. In this case, we can easily solve the LLG equation, Eq. (1). For simplicity of notation, we drop the subscripts $i$ and superscripts $c$, and remember that we are describing the precession of the $i$ th nanomagnet in the $c$ th crystal. We define the axes to describe the magnetization direction of this particular nanomagnet, $\hat{\boldsymbol{M}}, \hat{\boldsymbol{\theta}}$, and $\hat{\boldsymbol{\phi}}$, where $\hat{\boldsymbol{\phi}}=$ 
$\hat{\boldsymbol{M}} \times \hat{\boldsymbol{\theta}}$, and then write

$$
\begin{aligned}
\boldsymbol{B}^{\mathrm{eff}} & =B_{z} \hat{\boldsymbol{z}}=B_{z}(\hat{\boldsymbol{M}} \cos \theta-\hat{\boldsymbol{\theta}} \sin \theta) \\
& =\hat{\boldsymbol{M}} B_{M}+\hat{\boldsymbol{\theta}} B_{\theta} .
\end{aligned}
$$

Since the magnitude of the dipole moment $M_{s}$ is conserved, in spherical coordinates Eq.(1) leads to

$$
\begin{aligned}
\frac{d \hat{\boldsymbol{M}}}{d t} & =\hat{\boldsymbol{\theta}} \frac{d \theta}{d t}+\hat{\boldsymbol{\phi}} \sin \theta \frac{d \phi}{d t} \\
& =\hat{\boldsymbol{\theta}} \alpha B_{\theta}+\hat{\boldsymbol{\phi}} \gamma B_{\theta} .
\end{aligned}
$$

Finally, from

$$
\begin{aligned}
& \frac{d \theta}{d t}=-\alpha\left|\boldsymbol{B}^{\mathrm{eff}}\right| \sin \theta, \\
& \frac{d \phi}{d t}=-\gamma\left|\boldsymbol{B}^{\mathrm{eff}}\right|
\end{aligned}
$$

we obtain for a very small time interval $d t$,

$$
\begin{aligned}
\phi\left(t_{0}+d t\right) & \approx \phi\left(t_{0}\right)-\gamma\left|\boldsymbol{B}^{\mathrm{eff}}\left(t_{0}\right)\right| d t \\
\theta\left(t_{0}+d t\right) & \approx \theta\left(t_{0}\right)-\alpha\left|\boldsymbol{B}^{\mathrm{eff}}\left(t_{0}\right)\right| \sin \left[\theta\left(t_{0}\right)\right] d t .
\end{aligned}
$$

These equations were used in our numerical calculations. In order to relate the angles to measurable quantities, however, we note that it is possible to integrate Eqs. (14) and 15) exactly, obtaining

$$
\begin{aligned}
\theta(t)= & \cos ^{-1}\left[\operatorname { t a n h } \left(\operatorname { t a n h } ^ { - 1 } \left(\cos \left[\theta\left(t_{0}\right)\right]\right.\right.\right. \\
& \left.\left.+\alpha \int_{t_{0}}^{t} d \tau\left|B^{\mathrm{eff}}(\tau)\right|\right)\right] \\
\phi(t)= & \phi\left(t_{0}\right)-\gamma \int_{t_{0}}^{t} d \tau\left|\boldsymbol{B}^{\mathrm{eff}}(\tau)\right|
\end{aligned}
$$

which is equivalent to that obtained using a somewhat different technique. 3. We note that by expanding Eqs. (18) and 19) to leading order in $d t$, we recover Eqs. (17) and (16), respectively.

However, these more general forms for $\theta(t)$ and $\phi(t)$ lead to a more physical interpretation of our method. Since the dimensionless magnetization components along and perpendicular to $\boldsymbol{B}^{\mathrm{eff}}$ are $M_{z}=\cos \theta, M_{x}=$ $\sin \theta \cos \phi$, and $M_{y}=\sin \theta \sin \phi$, we have

$$
\begin{aligned}
& M_{z}(t)=\tanh \left(\tanh ^{-1}\left[M_{z}\left(t_{0}\right)\right]+\alpha \int_{t_{0}}^{t} d \tau\left|B^{\mathrm{eff}}(\tau)\right|\right) \\
& M_{x}(t)=\sqrt{1-\left[M_{z}(t)\right]^{2}} \cos [\phi(t)] \\
& \text { and }
\end{aligned}
$$

$$
M_{y}(t)=\sqrt{1-\left[M_{z}(t)\right]^{2}} \sin [\phi(t)] .
$$

Independent of the coordinates, we must assure that (for the $i$ th nanomagnet in the $c$ th configuration) $\boldsymbol{M}$ changes its direction smoothly, in order to obtain a reliable calculation for the overall $\overrightarrow{\mathcal{M}}$. Since each component of $\boldsymbol{M}$ cannot change dramatically, we must therefore require $\theta \ll 2 \pi$ and $\phi \ll 2 \pi$. These restrictions then require us to set the time integration step width $d t$ sufficiently small. If for example, $\gamma / \alpha$ were on the order of $10^{+11}$ and $\left|\boldsymbol{B}^{\mathrm{eff}}\right|$ were in the range $10^{-3}-10^{-2}$ $\mathrm{T}$, we would require $d t<10^{-11} \mathrm{~s}$. For sweep rate $\frac{\Delta B}{\Delta t} \approx 10^{-2} \mathrm{~T} / \mathrm{s}$, where $\Delta t=N_{t} d t \approx 10^{-4} \mathrm{~s}, N_{t}$ must be on the order of $10^{7}$. Since we would need to recalculate the direction of the magnetization of each nanomagnet $N_{t}$ times in each $\Delta B$ step, this would be a significant challenge with present day computers.

One thing we can do to make our calculations feasible for the sweep rates used in SMM studies is to set $\alpha$ extremely small, say $\alpha / \gamma \lesssim 10^{-10}$, although such small $\alpha$ values have not been reported in experiments. Otherwise, to study much larger but perhaps more reasonable $\alpha$ values, we would have to use much faster sweep rates, as in KS. 45

* Electronic address: alcantar@phys.ksu.edu

† Electronic address: klemm@phys.ksu.edu

‡ Electronic address: rahman@phys.ksu.edu

[1] D. Newns, W. Donath, G. Martyna, M. Schabes, and B. Lengsfield, J. Appl. Phys. 95, 3175 (2004).

[2] T. R. Koehler and D. R. Fredlin, IEEE Trans. Magn. 27, 4763 (1001).

[3] W. Chen, D. R. Fredkin, and T. R. Koehler, IEEE Trans. Magn. 29, 2124 (1993).

[4] J. F. Smyth, S. Schultz, D. R. Fredkin, D. P. Kern, S. A. Rishton, H. Schmid, M. Cali, and T. R. Koehler, J. Appl. Phys. 69, 5262 (1991).

[5] D. R. Fredkin and T. R. Koehler, IEEE Trans. Magn. 24, 2362 (1988).

[6] T. R. Koehler and D. R. Fredkin, IEEE Trans. Magn. 28, 1239 (1992).

[7] D. R. Fredkin and T. R. Koehler, J. Appl. Phys. 63, 3179 (1988).

[8] D. R. Fredkin and T. R. Koehler, J. Appl. Phys. 67, 5544 (1990).

[9] T. R. Koehler, Physica B 233, 302 (1997).

[10] T. R. Koehler and M. L. Williams, IEEE Trans. Magn. 31, 2639 (1995).

[11] E. D. Boerner and H. N. Bertram, IEEE Trans. Magn. 33, 3052 (1997).

[12] E. D. Boerner and H. N. Bertram, IEEE Trans. Magn. 34, 1678 (1998).

[13] J. M. Deutsch, T. Mai, and O. Narayan, Phys. Rev. E 71, 026120 (2005).

[14] K. Zhang and D. R. Fredkin, J. Appl. Phys. 87, 4795 (2000).

[15] M. N. Leuenberger and D. Loss, Nature (London) 410, 789 (2001).

[16] F. Luis, J. Bartolomé, J. F. Fernández, J. Tejada, J. M. Hernández, X. X. Zhang, and R. Ziolo, Phys. Rev. B 55, 11448 (1997); M. A. Novak and R. Sessoli, in Quantum Tunneling of the Magnetization-QTM'94, edited by L. 
Gunter and B. Barbara, NATO ASI Series E: Appl. Sci., (Kluwer, Dordrecht 1995) 301, 171.

[17] C. Sangregorio, T. Ohm, C. Paulsen, R. Sessoli, and D. Gatteschi, Phys. Rev. Lett. 784645 (1997).

[18] S. Hill, J. A. A. J. Perenboom, N. S. Dalal, T. Hathaway, T. Stalcup, and J. S. Brooks, Phys. Rev. Lett 80, 2453 (1998).

[19] S. M. J. Aubin, N. R. Dilley, M. B. Wemple, G. Christou, D. N. J. and Hendrickson, J. Am. Chem. Soc. 120, 839 (1998).

[20] L. Bokacheva, A. D. Kent, and M. A. Walters, Phys. Rev. Lett. 85, 4803 (2000).

[21] T. Ohm, C. Sangregorio, and C. Paulsen, Europhys. J. B 6, 595 (1998); J. Low Temp. Phys. 113, 1141 (1998).

[22] J. A. A. J. Perenboom, J. S. Brooks, S. Hill, T. Hathaway, and N. S. Dalal, Phys. Rev. B 58, 330 (1998).

[23] W. Wernsdorfer and R. Sessoli, Science 284, 133 (1999).

[24] D. V. Efremov and R. A. Klemm, Phys. Rev. B 66, 174427 (2002).

[25] R. Tiron, W. Wernsdorfer, D. Foguet-Albiol, N. AliagaAlcalde, and G. Christou, Phys. Rev. Lett. 91, 227203 (2003).

[26] M. Soler, W. Wernsdorfer, K. Folting, M. Pink, and G. Christou, J. Am. Chem. Soc. 126, 2156 (2004).

[27] W. Wernsdorfer, N. Aliaga Alcalde, D. Hendrickson, and G. Christou, Nature (London) 416, 406 (2002).

[28] J. Van Slageren, Introduction to Molecular Magnetism, http://www.pil.physik.uni-stuttgart.de

[29] J. R. Friedman, M. P. Sarachik, J. Tejada, and R. Ziolo, Phys. Rev. Lett. 76, 3830 (1996).

[30] L. Thomas, F. Lionti, R. Ballou, D. Gatteschi, R. Sessoli, and B. Barbara, Nature (London) 383, 145 (1996).

[31] W. Wernsdorfer, S. Bhaduri, R. Tiron, D. N. Hendrickson, G. Christou, J. Mag. Magn. Mat. 272, 1109 (2004).

[32] W. Wernsdorfer, S. Bhaduri, R. Tiron, D. N. Hendrickson, and G. Christou, Phys. Rev. Lett. 89, 197201 (2002).

[33] H. De Raedt, S. Miyashita, K. Michielsen, and M. Machida, Phys. Rev B 70, 064401 (2004).

[34] N. V. Prokof'ev and P. C. E. Stamp, Phys. Rev. Lett. 80, 5794 (1998).

[35] J. F. Fernández and J. J. Alonso, Phys. Rev. Lett. 91, 047202 (2003).

[36] I. S. Tupitsyn and P. C. E. Stamp, Phys. Rev. Lett. 92, 119701 (2004).

[37] J. F. Fernández and J. J. Alonso, Phys. Rev. Lett. 92, 119702 (2004).

[38] P. Santini, S. Carretta, E. Liviotti, G. Amoretti, P. Carretta, M. Filibian, A. Lascialfari, and E. Micotti, Phys. Rev. Lett. 94, 077203 (2005).
[39] Y. Furukawa, K. Kumagai, A. Lascialfari, S. Aldrovandi, F. Borsa, R. Sessoli, and D. Gatteschi, Phys. Rev. B 64, 094439 (2001).

[40] P. W. Anderson, Phys. Rev. 82, 342 (1951).

[41] H. Margenau and W. W. Watson, Rev. Mod. Phys. 8, 22 (1936).

[42] P. W. Anderson, Phys. Rev. 88, 1214 (1952).

[43] E. M. Lifshitz and L. P. Pitaevskii, Course of Theoretical Physics Vol.5: Statistical Physics (Pergamon, New York, 1980), Part 2.

[44] Huei Li Huang, Chin. J. Phys., 35 (6-II), 909 (1997).

[45] M. A. Kayali and W. M. Saslow, Phys. Rev. B 70, 174404 (2004).

[46] R. L. Stamps and R. E. Camley, Phys. Rev. B 60, 11694 (1999); Phys. Rev. B 60, 12264 (1999).

[47] J. F. Fernández and J. J. Alonso, Phys. Rev. B 62, 53 (2000); ibid. 65, 189901(E) (2002).

[48] M. Evangelisti, F. Luis, F. L. Mettes, N. Aliaga, G. Aromí, J. J. Alonso, G. Christou, and L. J. de Jongh, Phys. Rev. Lett. 93, 117202 (2004).

[49] R. K. Wangsness and F. Bloch, Phys. Rev. 89, 728 (1953).

[50] D. R. Fredkin and A. Ron, Phys. Rev. B 61, 8654 (2000).

[51] J. Ho, F. C. Khanna, and B. C. Choi, Phys. Rev. B 70, $172402(2004)$.

[52] J. Ho, F. C. Khanna, and B. C. Choi, Phys. Rev. Lett. 92, 097601 (2004).

[53] J. Sinova, T. Jungwirth, X. Liu, Y. Sasaki, J. K. Furdyna, W. A. Atkinson, and A. H. MacDonald, Phys. Rev. B 69, 085209 (2004).

[54] M. Alcántara Ortigoza, R. A. Klemm, and T. S. Rahman (unpublished).

[55] E. Z. Meǐlikhov and R. M. Farzetdinova, J. Exp. Theor. Phys., 98, 1367 (2004).

[56] W. Wernsdorfer and R. Sessoli, Science 284, 133 (1999).

[57] Y. Shapira, M. T. Liu, S. Foner, C. E. Dubé, and P. J. Bonitatebus, Jr., Phys. Rev. B 59, 1046 (1999).

[58] Y. Shapira, M. T. Liu, S. Foner, R. J. Howard, W. H. Armstrong, Phys. Rev. B 63, 094422 (2001).

[59] D. V. Efremov and R. A. Klemm, Proceedings of the 24th International Conference on Low Temp. Phys., (in press); cond-mat/0409168 (unpublished).

[60] A. Morello, F. L. Mettes, F. Luis, J. F. Fernández, J. Krzystek, G. Aromí, G. Christou, and L. J. de Jongh, Phys. Rev. Lett. 90, 017206 (2003).

[61] Y. Takagaki and K. H. Ploog, Phys. Rev. B 71, 184439 (2005). 